\title{
Collaborative Learning at CSCL 2013
}

\author{
Gerry Stahl • Nancy Law $•$ Friedrich Hesse
}

Published online: 9 August 2013

(C) International Society of the Learning Sciences, Inc. and Springer Science+Business Media New York 2013

In Madison, Wisconsin, USA, with its Northern European heritage, collaborative learning is fueled by brats and beer. At the international CSCL conference in June, there were many formal and informal opportunities to build knowledge about our field with colleagues from around the world while sipping drinks on the shores of the sunny lakes, along the vibrant college-town avenues, or in the university halls.

The intense week of interaction began - at least for some - with a daylong retreat of the ISLS Board. Discussion focused on plans for increasing the impact of the CSCL and Learning Sciences research community globally. A more interactive website is imminent and increased outreach to communities that are just discovering CSCL is planned. Efforts to increase access to the contents of the journals - ijCSCL and $J L S$ - as well as conference papers and the CSCL book series are underway.

The pre-conference began the next day with a variety of well-attended workshops. One was on interactional resources spanning multiple levels of analysis in CSCL settings-as discussed in the three preceding issues of $i j C S C L$. Another of especially general interest was a workshop on creating a cyber-infrastructure that can support engagement by multiple researchers in working toward answers to important theory-driven research questions for design-based research. As always, the doctoral consortium and early career workshops were valuable for the many mentors as well as the participants.

The following day began with half-day workshops. We three attended one that seemed particularly promising for the field of CSCL. The international PISA test-which rates student math, science, and reading skills in over 70 countries around the world-is planning to introduce measures of collaborative problem-solving skills in 2015. This could mean that students, parents, teachers, schools, and policy makers in many countries will urgently want to know about collaborative learning. In fact, the test will be computer supported, having students tested through interacting with a computer system. This workshop was the first time

G. Stahl $(\bowtie)$

Drexel University, Philadelphia, PA, USA

e-mail: Gerry@ijCSCL.org

N. Law

University of Hong Kong, Hong Kong, People's Republic of China

e-mail: Nancy@ijCSCL.org

F. Hesse

Knowledge Media Research Center (KMRC), Tubingen, Germany

e-mail: Friedrich@ijCSCL.org 
that the framework for measuring collaborative problem solving in the PISA tests was made public. Fortunately, a number of CSCL and Learning Sciences researchers are closely involved in this effort and are committed to making the process as public as possible. The debates and discussion during the workshop raised important theoretical and methodological challenges to measuring collaborative problem solving, and there is general agreement that such open communication between the CSCL and the psychometric communities is beneficial to advances in both large-scale assessment methodologies as well as in our understanding of collaborative learning and problem solving. We welcome submissions to ijCSCL on the PISA approach and its implications for CSCL.

Highlights of the conference included three keynote presentations, each of which broadened the discussions of CSCL. They presented insights into behaviors related to collaborative learning among chimpanzees who live in the present only, teenagers who build rapport by insulting each other, and young girls who express themselves in amateur videos. Another highlight was the invited presidential symposium, organized by ISLS current president, Frank Fischer, in-coming president, Cindy Hmelo-Silver, and former president, Susan Goldman. Because of its potential interest to the ijCSCL readership, we present a summary of the symposium presentations below.

Of course, the heart of the conference was the presentation of lecture papers, interactive papers, posters, demos, symposia, etc. - too many for anyone to attend all of them. We hope to publish extended journal versions of some of this important research next year. For this issue, we present a mix of empirical, pedagogical and theoretical papers addressing current topics in CSCL.

\section{Looking back and looking ahead: Twenty international years of CSCL}

The Presidential Symposium presenters analyzed the research and the development of the CSCL community in their respective regions of the world. Gerry Stahl presented a brief history of CSCL in North America; Paul Kirschner presented his view on CSCL research and communities in Europe; Peter Reimann analyzed CSCL research in Australia; and Nancy Law contributed her perspective on CSCL in Asia. In the role of the discussant, conference program co-chair Nikol Rummel provided a synthesizing perspective on historical and future trends.

Almost two decades after the first conference on computer support for collaborative learning, four contributors analyzed the research and the development of the CSCL community in their respective regions of the world. Questions they addressed included the following: What were the origins and early stages of CSCL in this area of the world? What have been important research questions, concepts, and methods? Which unique contributions to CSCL research have there been from this area of the world? What have been the role and the relation of different disciplines within CSCL research, e.g., computer science, psychology, and educational sciences? Looking ahead, what future trajectories can be expected and what would be desirable futures of CSCL research? The discussant reflected on the presence in the current conference of the trends presented in this symposium.

For many of the leading early North American CSCL researchers, the goal was to use CSCL innovations as levers to transform education by promoting collaborative learning. They investigated the interaction within the group and the group processes related to social dynamics as well as to knowledge building. Research addressed aspects like design of technology, analysis of collaborative learning, and the evaluation of collaborative-learning outcomes. A major contribution of North America to CSCL research has been the emphasis 
on design-based research, in which iterative cycles of trials in realistic settings are used to drive design of technology and pedagogy. Future trends in CSCL research are toward increasing international collaborations and projects.

The hallmark of European CSCL is its diversity. Across Europe, research groups are designing tools for CSCL and studying their implementation in terms of duration, scripting, and social dynamics. Aided by national, transnational and European programs, Europeans regularly work with and meet with each other, learning from each other in Networks of Excellence and European schools. The future promises continued cross-national efforts.

The main "driving" discipline behind CSCL research in Australia is applied computer science, in particular in the form of technology developments in higher education. Australia's innovative contributions to CSCL currently include tabletop computing in support of co-located, synchronous group work and group learning, collaborative webbased video annotation, and collaborative (academic) writing as a form of CSCL. Likely trajectories for future research are those focused on media-rich (synchronous) collaboration, including video conferencing and collaboration in immersive environments; increasing use of learning analytics in the context of CSCL studies, and studies into collaboration processes in design teams and virtual design studios.

CSCL research in Asia was stimulated in the 1990s by the formulation of IT master-plans in a number of countries and focused on improving education system-wide and preparing citizens for the 21 st century. There is strong interest in linking research and practice and an orientation toward collaborative knowledge building. The CSCL research conducted in Asia has a strong emphasis on pedagogy and assessment, bringing with it the challenge of integrating CSCL into the daily instructional milieu. A second emphasis in Asia is on teacher learning and professional teacher networks for knowledge building. Looking to the future, CSCL in Asia needs to take up the challenges inherent in research on CSCL at individual, group, and community levels.

Two themes emerge from considering the perspectives presented from the different regions of the world on the past and future of the field of CSCL: diversity and unity. On the one hand, we see a lot of diversity in the ways CSCL research has developed and is currently enacted. Differences concern, for instance, research foci (i.e., which letter of the CSCL acronym research focuses on), research methodology, overarching goals of the research, and the extent to which various stakeholder groups (e.g., learners, teachers, policy makers) guide the work or are considered. On the other hand, it is evident that there is unity in the diversity: The joint goal of the international community of CSCL is to make an impact on the way collaborative learning is implemented, both in terms of educational practice and policy. Developing the CSCL community as part of the larger umbrella of ISLS will be instrumental to enabling the CSCL community to have a say in international developments, such as the planned inclusion of the area of collaborative problem solving in PISA 2015. 